\title{
A new case of pragmatically deviant embedded disjunctions*
}

\author{
Milica Denić \\ $L S C P \& I J N$, \\ ENS-EHESS-CNRS, \\ PSL University
}

\begin{abstract}
In this paper, we discuss a novel observation that sentences such as '\#Each of these three girls is Mary, Susan, or Jane' are deviant. Its deviance is surprising: the sentence should convey that one of the three girls is Mary, another one of them is Susan, and yet another one is Jane; however, it cannot be naturally used to do so. We will propose that the deviance is caused by ignorance inferences which contradict common knowledge. If the proposal is on the right track, ignorance inferences need to be derived blindly to common knowledge, similarly to what has been proposed for scalar implicatures by Magri (2009).
\end{abstract}

Keywords: pragmatic deviance, obligatory implicatures, ignorance inferences, disjunction, contextual blindness

\section{Puzzle}

When disjunctions of definite noun phrases are embedded in the scope of a universal quantifier, the result is sometimes unexpectedly deviant. The deviance depends on the predicate that embeds the disjunction. For instance, when the predicate in question is the identity copula as in (1) or the predicate to write in (3), the result is deviant. When the predicate in question is minimally different, as the predicate to be called in (2) or the predicate to read in (4), the result is acceptable.

(1) (Context: Peter invited three girls to the party.)

\#Each of those three girls is Mary, Susan, or Jane.

(2) (Context: Peter invited three girls to the party.)

Each of those three girls is called Mary, Susan, or Jane.

* I am especially grateful to Emmanuel Chemla, Gennaro Chierchia, and Benjamin Spector for extremely helpful discussions and suggestions. Thanks a lot also to Amir Anvari, Moshe E. Bar-Lev, Brian Buccola, Rachel Dudley, Émile Enguehard, Danny Fox, Manuel Križ, Jeremy Kuhn, Mora Maldonado, and Philippe Schlenker, as well as the audience of SALT 28 for very helpful feedback. The research leading to this work was supported by ANR-17-EURE-0017.

(C2018 Denić 
A new case of pragmatically deviant embedded disjunctions

(3) (Context: Tolstoy, Zola and Rowling are great writers.)

\#Each of those three writers wrote Anna Karenina, Germinal, or Harry Potter.

(4) (Context: Ann, John, and Bob are great students.)

Each of those three students read Anna Karenina, Germinal, or Harry Potter.

To see why the deviance of (1) and of (3) is surprising, note that (1) is contextually equivalent to (8), assuming that it is common knowledge that Mary, Susan, and Jane have to be three different individuals (see (5), (6), and (7) for definitions of common knowledge, context set and contextual equivalence). Likewise, (3) is contextually equivalent to (9), assuming that it is common knowledge that for any book there can be exactly one singular or plural individual who wrote it ${ }^{1}$. Yet, surprisingly, (1) cannot be naturally used to do convey the meaning of (8), and neither can (3) to convey the meaning of (9). The main goal of this paper will be to try to understand why this is the case.

\section{(5) Common knowledge:}

A proposition $\phi$ is commonly known to a group of individuals if and only if all individuals in the group know that $\phi$, all know that all know it, all know that all know that all know it, etc. (Stalnaker 2002)

(6) Context set:

Context set is the set of possible worlds in which all the propositions that are common knowledge between the interlocutors are true.

(7) Contextual equivalence:

Two sentences $A$ and $B$ are contextually equivalent iff they have the same truth value in all the worlds of the context set.

(8) One of those three girls is Mary, another one is Susan, and yet another one is Jane.

(9) One of those three writers wrote Anna Karenina, another one wrote Germinal, and yet another one wrote Harry Potter.

Note that the deviance observed in (1) and (3) is not specific to each: the pattern is the same with every and all. When the disjunction is an argument to the copula as in (10) or to the predicate to write as in (12), the resulting sentence is deviant. When the disjunction is an argument to the predicate to be called as in (11) or to the predicate to read as in (13), the resulting sentence is acceptable.

1 In the case of co-authorship there would be exactly one plural individual who wrote the book. 
(10) a. \#Every one of those three girls is Susan, Mary, or Jane.

b. \#All of those three girls are Susan, Mary, or Jane.

(11) a. Every one of those three girls is called Susan, Mary, or Jane.

b. All of those three girls are called Susan, Mary, or Jane.

a. \#Every one of those three writers wrote Anna Karenina, Germinal, or Harry Potter.

b. \#All of those three writers wrote Anna Karenina, Germinal, or Harry Potter. (deviant under distributive reading)

a. Every one of those three students read Anna Karenina, Germinal, or Harry Potter.

b. All of those three students read Anna Karenina, Germinal, or Harry Potter.

The observed deviance is also not limited to universally quantified noun phrases; see for instance (14). However, for the simplicity of exposition, we will focus on the disjunction embedded in the scope of a universal quantifier; the main ideas that will be presented should carry over to the cases in (14) without major additional assumptions.

a. \#These three girls are Mary, Susan, or Jane.

b. \#Three of those girls are Mary, Susan, or Jane.

\section{Descriptive generalizations}

\subsection{Left-uniqueness}

We have observed the deviance of an embedded disjunction with certain predicates, such as the identity copula or to write, but not with others, such as to be called or to read. Which property makes a predicate pattern with one group or the other? For reasons that will become clear in Section 3, we will propose that to write and the identity copula pattern together because, when their internal argument is provided in sentences such as (15a) and (15b), given world knowledge, they can only be true of a unique (singular or plural) individual.

In other words, given world knowledge, the predicates to write (when its internal argument is from a domain of books) and the identity copula (when its internal argument is from a domain of individuals) have the property in (16), which we will refer to as left-uniqueness.
a. That girl is Mary.
b. That writer wrote Anna Karenina. 
A new case of pragmatically deviant embedded disjunctions

A predicate $P$ is left-unique iff

$\forall y$ in the relevant domain $\forall x[P(x, y) \Rightarrow \forall z[P(z, y) \Rightarrow z=x]]$

We propose as a descriptive generalization that if a predicate $P$ is left-unique when its internal argument is from some relevant domain $D$, then $P$ will pattern with the predicates to write or the identity copula; otherwise it will pattern with to read or to be called.

To see that the identity copula (when its internal argument is from a domain of individuals) and to write (when its internal argument is from a domain of books) are left-unique but not the predicates to be called (when its internal argument is from a domain of names) and to read (when its internal argument is from a domain of books), observe that the continuations in (17a) and (17c) sound contradictory, but not in (17b) and in (17d).

a. This girl is my sister Susan. \#That other girl is my sister Susan too.

b. This girl is called Susan. That other girl is called Susan too.

c. John wrote this book. \#Peter wrote this book too.

d. John read this book. Peter read this book too.

Let us see some further empirical support that left-uniqueness is indeed relevant for the deviance observed in (1) and (3).

First, consider the possibility/necessity modal contrast in (18). When the epistemic necessity modal is embedded in between the universal quantifier and the disjunction of the sentence (1), as in (18a), the result is deviant. The minimally different $(18 \mathrm{~b})$ with the epistemic possibility modal instead of the necessity modal is acceptable.

a. \#Each of these three people must be Mary, Susan, or Jane.

b. Each of these three people might be Mary, Susan, or Jane.

Crucially, when the identity copula is embedded under the necessity modal it is left-unique (cf. (19b)). When the identity copula is embedded under the possibility it is not left-unique. (cf. (19a)):

a. This girl must be John's sister Mary. \#That other girl must be John's sister Mary too.

b. This girl might be John's sister Mary. That other girl might be John's sister Mary too.

Second, consider a minimal pair of predicates such as to be a mother/father of vs. to be a brother/sister of, when their internal argument is from a domain of individuals. The predicates to be a mother/father of pattern together with the identity 
copula and to write both with respect to the deviance and to the left-uniqueness (cf. (20a) and (21a)). The predicates to be a brother/sister of pattern together with to be called and to read both with respect to the deviance and to the left-uniqueness (cf. (20b) and (21b)).

a. \#Each of these three men is Peter's, John's or Mary's father.

b. Each of these three boys is Peter's, John's or Mary's brother.

(21) a. That man is Peter's father. \#That other man is Peter's father too.

b. That boy is Peter's brother. That other boy is Peter's brother too.

Finally, consider what happens when the internal argument of to write is not from a relevant domain for it to be left-unique. For instance, when its internal argument is from a domain of letters of the alphabet, the predicate to write is not left-unique, and note that (22), which is structurally similar to (1) and (3), is not deviant (it could perfectly be used in a situation in which, for instance, each of John's three students wrote a number of letters on the board):

(22) Each of John's three students wrote the letter A, the letter D, or the letter K on the board.

\subsection{Domain alternatives}

The fact that there is a disjunction in (1) and (3) has an interesting consequence. Consider the alternatives of (1) and (3) obtained by dropping a proper subset of disjuncts. Following the terminology introduced in Chierchia 2013 we will refer to this type of alternatives as domain alternatives. For instance, in examples (23) and (24), the (b) sentences are examples of domain alternatives of the (a) sentences.

a. \#Each of these three people is Mary, Susan, or Jane.

b. \#Each of these three people is Susan.

a. \#Each of these three writers wrote Anna Karenina, Germinal, or Harry Potter.

b. \#Each of these three writers wrote Anna Karenina.

Note then that the (b) sentences in (23) and (24) are themselves deviant. This is because, due to the left-uniqueness of the identity copula and to write, they are contextual contradictions, in the following sense:

\section{Contextual contradiction:}

A sentence is a contextual contradiction iff it is false in all of the worlds in the context set. 
A new case of pragmatically deviant embedded disjunctions

Consider now domain alternatives of minimally different sentences with predicates which are not left-unique. In examples (26) and (27), the (b) sentences are examples of domain alternatives of the (a) sentences. This time, (b) sentences in (26) and (27) are not deviant (they are not contextual contradictions).
a. Each of these three girls is called Mary, Susan, or Jane.
b. Each of these three girls is called Mary.
a. Each of these three students read Anna Karenina, Germinal, or Harry Potter.
b. Each of these three students read Anna Karenina.

\section{Proposal}

The account that will be put forward in what follows builds on the observation that domain alternatives of pragmatically deviant sentences in (1) and (3) are contextual contradictions, while the domain alternatives of minimally different non-deviant (2) and (4) are not.

More specifically, we will propose that because their domain alternatives are contextual contradictions, the sentences such as (1) and (3) trigger ignorance inferences which themselves are contextual contradictions, ultimately leading to pragmatic deviance of (1) and (3).

Let us first see what ignorance inferences are on a sentence with unembedded disjunction in (28a). (28a) has the domain alternatives in (28b). (28a) is usually interpreted with the ignorance inference in (28c): in other words, (28a) triggers the ignorance inference about its domain alternatives (Gazdar 1980).
a. John is in Paris or in London.
b. John is in Paris; John is in London
c. $\rightsquigarrow$ The speaker is ignorant about whether or not John is in Paris and he is ignorant about whether or not John is in London.

Consider now what happens if (1) and (3), repeated here as (29a) and (30a), trigger ignorance inferences about their domain alternatives. These ignorance inferences are paraphrased in (29b) and (30b). One can immediately see the problem with these ignorance inferences: given that the domain alternatives of (29a) and (30a) are contextual contradictions, as argued in Section 2, the speaker cannot possibly be ignorant about them - he must know that the domain alternatives are false. The ignorance inferences in (29b) and (30b) are thus themselves contextual contradictions. We propose then that the sentences (29a) and (30a) are deviant because they trigger ignorance inferences in (29b) and (30b), and these inferences are contextual contradictions. 
a. \#Each of these three girls is Mary, Susan, or Jane.

b. The speaker is ignorant about whether each of these three girls is Mary and he is ignorant about whether each of these three girls is Susan and he is ignorant about whether each of these three girls is Jane.

a. \#Each of these three writers wrote Anna Karenina, Germinal, or Harry Potter.

b. The speaker is ignorant about whether each of these three writers wrote Anna Karenina and he is ignorant about whether each of these three writers wrote Germinal and he is ignorant about whether each of these three writers wrote Harry Potter.

Let us summarize why (1) and (3) are deviant according to the proposal. Given that the identity copula and the predicate to write are left-unique when their internal argument is from a domain of individuals and from a domain of books respectively, the domain alternatives of (1) and (3) are contextual contradictions. This in turn entails that the speaker cannot be ignorant about them. (1) and (3) are deviant because they trigger ignorance inferences about their domain alternatives, and these inferences themselves are contextual contradictions.

\section{Ignorance inferences of embedded disjunction}

The crucial hypothesis of the proposal put forward in Section 3 is that sentences such as (1) and (3) trigger ignorance inferences with respect to their domain alternatives. In this section we check whether this is a viable hypothesis because while sentences with unembedded disjunctions are known to trigger ignorance inferences, sentences with embedded disjunction usually don't. For instance, (31a) usually triggers the inferences in (31b), which have been called in the literature alternatively free choice inferences or distributive inferences (Spector 2006; Fox 2007; Klinedinst 2007, see experimental data in Crnič, Chemla \& Fox 2015.) Importantly, if (31a) triggers the inferences in (31b), it cannot trigger the ignorance inferences about domain alternatives in (31c) - in this particular example, the inferences in (31b) are inconsistent with the inferences in (31c).

\section{a. All 20 of my closest friends are (either) French or Spanish.}

b. $\rightsquigarrow$ At least one of my 20 closest friends is French.

$\rightsquigarrow$ At least one of my 20 closest friends is Spanish.

c. $\quad \Varangle \rightarrow$ The speaker is ignorant about whether or not all 20 of his closest friends are French.

$\not \rightarrow$ The speaker is ignorant about whether or not all 20 of his closest friends are Spanish. 
A new case of pragmatically deviant embedded disjunctions

We will now show that despite the fact that (31a) does not trigger ignorance inferences about its domain alternatives, there is evidence that sentences such as (1) and (3) do. Consider what happens when the domain of the universal quantifier has a very small cardinality, as in (32a). Strikingly, the inference pattern is reversed. (32a) is reported to strongly trigger the ignorance inferences in (32c) instead of the distributive inferences in (32b):

a. Both of my closest friends are (either) French or Spanish.

b. $\quad \psi \rightarrow$ At least one of my two closest friends is French.

$\not \rightarrow$ At least one of my two closest friends is Spanish.

c. $\rightsquigarrow$ The speaker is ignorant about whether or not both of his closest friends are Spanish.

$\rightsquigarrow$ The speaker is ignorant about whether or not both of his closest friends are French.

The only difference between (31a) and (32a) is the cardinality of the domain of the universal quantifier: twenty in (31a) and two in (32a). Yet this property seems to somehow matter for whether the universally quantified sentences with embedded disjunction trigger ignorance inferences about their domain alternatives. More accurately, it is not merely the cardinality of the domain of the universal quantifier that determines whether a sentence with embedded disjunction triggers ignorance inferences: rather, what seems to be relevant is the ratio between this cardinality and the number of disjuncts. For instance, (33a) is reported to be more easily interpreted without ignorance inferences than (33b).

a. All four of my closest friends are French or Spanish.

b. All four of my closest friends are French, Spanish, German, or Portuguese.

We will not provide here an account for this observation (see Denić 2018 for one possible account). However, what is crucial for current purposes is that the basic deviant examples, (1) and (3), have the same (1:1) ratio between the cardinality of the domain of the universal quantifier and the number of disjuncts as the sentences which have been identified to strongly trigger ignorance inferences, namely the sentences in (32a) and (33b).

Hence, there is evidence that sentences which share relevant properties with (1) and (3) trigger ignorance inferences, making the proposal in Section 3 a viable one. 


\section{Blind ignorance inferences}

According to the proposal in Section 3, (1) and (3) are deviant because they trigger ignorance inferences which contradict common knowledge. This however assumes that implicatures generally, and ignorance inferences specifically, are derived blindly from common knowledge. The idea that implicatures are derived blindly from common knowledge has been initially put forward in Magri 2009 for the case of scalar implicatures. The pragmatically deviant (34) is an example of the cases that motivated this proposal.

\#Some Italians come from a warm country.

Simplifying a lot, Magri's (2009) proposal for why (34) is deviant is that (34) activates as its scalar alternative (35a), and thus it has as its scalar implicature the negation of (35a), paraphrased in (35b). According to Magri's proposal, (34) is deviant because the conjunction of (34) and (35b) contradicts common knowledge.

a. All Italians come from a warm country.

b. Not all Italians come from a warm country.

Note that in general blind derivation of implicatures is easier to accommodate within theories according to which implicatures are derived in grammar, such as exhaustification-based approaches (Fox 2007; Chierchia, Fox \& Spector 2008), rather than with neo-Gricean views on ignorance implicatures derivation (Sauerland 2004). It has already been proposed that ignorance inferences are derived in grammar (Meyer 2013, 2014) - the phenomenon discussed in this paper could thus be an argument for this type of view on ignorance implicatures.

\section{Optionality of implicatures}

Magri's proposal that (34), repeated here as (36a), is deviant because of the scalar implicature it triggers faces an immediate challenge in that scalar implicatures are known to be optional. The current proposal that (1) and (3), repeated here as (36b) and (36c), are deviant because they trigger ignorance inferences which contradict common knowledge inherits the same challenge. We will comment more in this section on what is at stake.

a. \#Some Italians come from a warm country.

b. \#Each of these three girls is Mary, Susan, or Jane.

c. \#Each of these three writers wrote Anna Karenina, Germinal, or Harry Potter. 
A new case of pragmatically deviant embedded disjunctions

The optionality of scalar implicatures is demonstrated by the fact that the discourse in (37) is coherent.

Some of my students are French. In fact, they all are.

We have also seen that some cases of embedded disjunction, such as (38), are easily interpreted without ignorance inferences.

(38) Each of these 20 girls is called Mary, Susan, or Jane.

The challenge is this: if scalar implicatures and ignorance inferences are optional, how come that we ever perceive the sentences such as those in (36) as deviant?

Magri proposes an account according to which the scalar implicature is necessarily derived in (36a), while it is not necessarily derived in cases such as (37). He adopts an exhaustification-based framework for implicature derivation (Fox 2007; Chierchia et al. 2008), together with the theory of alternatives as in Katzir 2007; Fox \& Katzir 2011. Simplifying a lot, he proposes that the exhaustifying operator exh, defined in (39), is present at every scope site, but that the scalar alternative cannot be pruned in cases such as (36a) (pruned alternatives are those alternatives which are left out of the alternative set when the implicatures are calculated), while it can be pruned in cases such as (37). Alternatives are usually considered to be prunable due to relevance considerations: when an alternative is irrelevant, it can be left out of the alternative set (for discussion on constraints for alternative pruning, see Katzir 2007 and Fox \& Katzir 2011).

a. $\quad \operatorname{Exh}(p)=p \wedge \bigwedge_{q \in I E(p)} \neg q$

b. $\quad \operatorname{IE}(\mathrm{p})=\bigcap\left\{\mathrm{A}^{\prime} \subseteq \operatorname{ALT}(\mathrm{p}): \mathrm{A}^{\prime}\right.$ is a maximal set in $\mathrm{ALT}(\mathrm{p})$ which can be negated consistently with $\mathrm{p}\}$

Even though we are not in a position to provide an account for why ignorance inferences are obligatory with (36b) and (36c), but not with (38), here is a possible strategy. Consider first what alternatives sentences such as (36b) and (38) activate. There is a debate in the literature building on structurally defined alternatives (Katzir 2007; Fox \& Katzir 2011) as to whether sentences of the form as in (40a), with A and $\mathrm{B}$ being predicates of type $\langle e, t\rangle$, activate only the alternatives in (40b) or both the alternatives in (40b) and in (40c) (see also Bar-Lev \& Fox 2017, fn. 7).

a. Everyone is A or B.

b. (i) Everyone is A, (ii) Everyone is B, (iii) Everyone is A and B

c. (iv) Someone is A, (v) Someone is B, (vi) Someone is A or B, (vii) Someone is A and B

Under exhaustification-based account of implicature derivation, if (40a) activates 
only the alternatives in (40b), then all of its alternatives are innocently excludable (IE) (cf. (39b)). Negating all of the alternatives in (40b), together with asserting the prejacent, derives the distributive inferences in (41a), but not the ignorance inferences in (41b).

a. Someone is A and someone is B.

b. The speaker is ignorant about whether everyone is $\mathrm{A}$ and he is ignorant about whether everyone is B.

On the other hand, if (40a) activates both (40b) and (40c) as its alternatives, then the only IE alternatives are (iii) and (vii) from (40b) and (40c) respectively. Crucially, as the alternatives (i) and (ii) from (40b) are no longer IE, we will not derive distributive inferences. Rather, assuming that ignorance inferences are derived about all the alternatives whose truth is not settled (see Buccola \& Haida 2018 and references therein), we will derive ignorance inferences about (i), (ii), (iv) and (v) from (40b) and (40c). In other words, we will derive the ignorance inference in (41b) but not the distributive inferences in (41a).

Therefore, in order to capture that ignorance inferences are not necessarily triggered by (38) but that they are necessarily triggered by (36b) and (36c), one possible strategy would be to provide constraints on alternative pruning that are sensitive to the domain size of the universal quantifier which is such that it allows pruning of alternatives in (40c) for sentences like (38), but not for sentences such as (36b) and (36c) (for further details, see Denić 2018).

\section{Empirical challenges}

There are four main empirical points that the account proposed here cannot immediately capture. Nonetheless, it might be extended in order to do so, and we point out to potential ways of achieving that.

\subsection{Modal contrast}

In order to capture the modal contrast between (42a) and (43a) along the same lines as we have explained the deviance of (1) and (3), we would have to assume that (42a) and (42b) have as ignorance inferences respectively (42b) and (43b).

(42) a. Each of these three people might be Mary, Susan, or Jane.

b. The speaker is ignorant about whether each of these three people might be Susan.

(43) a. \#Each of these three people must be Mary, Susan, or Jane. 
A new case of pragmatically deviant embedded disjunctions

b. The speaker is ignorant about whether each of these three people must be Susan.

Note that, as both might in (42a) and must in (43a) are epistemic modals, the inferences in (42b) and (43b) can be informally paraphrased respectively as (44a) and (44b).

a. The speaker is ignorant about whether for each of these three girls there is a world epistemically accessible to him in which she is Susan. (likewise, Mary, Jane...)

b. The speaker is ignorant about whether for each of these three girls, in all of the worlds epistemically accessible to him, she is Susan. (likewise, Mary, Jane...)

This means that, if indeed (42a) and (43a) trigger ignorance inferences about domain alternatives as in (42b) and (43b), these would have to be inferences according to which the speaker is ignorant about his own epistemic states. As an epistemic state such that in all possible worlds each of the three girls is Mary is in fact an impossible epistemic state given common knowledge, the speaker cannot be ignorant about whether or not this is his epistemic state. Hence the deviance of (43). On the other hand, as the epistemic state which is such that for each of the three girls there is a possible world in which she is Mary is a possible epistemic state, the speaker can in principle, in a strange state of uncertainty about what it is that he knows, be ignorant about whether he is in this state.

This line of explanation is, however, problematic. The reason is that the epistemic necessity modals are usually thought to satisfy positive and negative introspection: in other words, if a person $x$ believes a proposition $p, x$ believes that $x$ believes $p$, and if $x$ does not believe $p, x$ believes that $x$ does not believe $p$ (see Kaufmann, Condoravdi \& Harizanov 2006 and references therein). Resolving this issue is left for future work.

\subsection{Larger domain examples}

Consider the deviant sentence in (45a). One of its domain alternatives, in (45b), is a contextual contradiction, and the other one, in (45c) is not.

a. \#Each of the seven girls in this photo is my daughter Lisa or a cousin of mine.

b. \#Each of the seven girls in this photo is my daughter Lisa.

c. Each of the seven girls in this photo is a cousin of mine.

If the sentence in (45a) triggered ignorance inferences about its domain alternatives, 
we could explain its deviance in the same way as we did for the sentences (1) and (3). However, while we provided independent evidence that sentences such as (1) and (3) should trigger ignorance inferences in Section 4, such evidence is lacking for (45a). To see why, consider (46), which is structurally similar to (45a). It is quite natural to interpret (46) with the distributive inferences in (46b) and without the ignorance inferences in (46c). Hence, we do not have an argument that (45a) should be deviant because of its ignorance inferences.

a. Each of the seven girls in my class is from France or from a country in South America.

b. $\quad \rightsquigarrow$ At least one of the seven girls in my class is from France.

$\rightsquigarrow$ At least one of the seven girls in my class is from a country in South America.

c. $\quad \Varangle \rightarrow$ The speaker is ignorant about whether or not each of the the seven girls is from France.

$\not \rightarrow$ The speaker is ignorant about whether or not each of the the seven girls is from a country in South America.

We would like to point to an alternative approach for the deviance of (45a), which is nonetheless in the same spirit as the current proposal. Spector (2018) observes that sentences such as (46a) trigger not only the distributive inferences in (46b), but also an inference about how many of the seven girls approximately are from France, and how many from a country in South America (we will refer to this in the continuation as the distribution estimate inference). The content of this inference for a sentence such as (46a) seems to be that there is approximately as many of the seven girls who are from France as there is of those who are from South America.

If this distribution estimate inference is blind to common knowledge too, we can explain the deviance of (45a). In other words, (45a) would be deviant because it triggers the distribution estimate inferences in (47).

Approximately the same number of the girls in the photo are my daughter Lisa as the number of girls in the photo who are cousins of mine.

Note that if this explanation for the deviance of (45a) is indeed on the right track, many different linguistic inferences (and not only scalar implicatures and ignorance inferences) would have to be derived blindly to common knowledge. Whether this is empirically desirable is to be seen in future research. 
A new case of pragmatically deviant embedded disjunctions

\subsection{Downward-entailing contexts}

We have so far not discussed what happens when sentences such as (1) and (3) are embedded under a downward-entailing operator. (48) is an example that illustrates this. The intuitions about (48) seem to be less stable, but at least some speakers find the sentence deviant.

(48) ?\#It's not the case that both of these girls are Susan or Jane.

If we wanted to explain the deviance of (48) along the same lines as above, we would have to say that (48) is deviant because it triggers the ignorance inferences in (49).

a. The speaker is ignorant about whether or not both of these girls are Susan.

b. The speaker is ignorant about whether or not both of these girls are Jane.

There is however a problem with this. First, a structurally similar sentence in (50a) triggers the scalar inference in (50b), but it clearly does not trigger ignorance inferences in (50c). In fact, a speaker cannot simultaneously believe (50a) and be ignorant about whether or not both of his students got an A (and whether or not both of his students got a B): therefore, if upon hearing (50), one inferred (50c), one would also need to infer that the speaker doesn't believe his own utterance (50a) and thus violates the Gricean maxim of quality (Grice 1975).

a. It's not the case that both of my students got an A or a C.

b. $\rightsquigarrow$ One of my students got an A or a C.

c. $\quad \Varangle \rightarrow$ The speaker is ignorant about whether or not both of his students got an A.

$\not \rightarrow$ The speaker is ignorant about whether or not both of his students got a $\mathrm{C}$.

We thus have a problem for the proposal put forward in this paper. It is important to note, however, that the situation is somewhat similar with the sentences discussed in Magri (2009). Note that (51), in which the quantifier some Italians is in a downward-entailing environment, is deviant, just like (34), repeated here in (52).

(51) \#It's not the case that some Italians come from a cold country.

(52) \#Some Italians come from a warm country.

Note that the reason for deviance of (51) cannot be that it triggers the implicature in (53) - even if (51) triggered the implicature in (53), this implicatures wouldn't be in any way contradictory with common knowledge. 
One possible way to explain the deviance of (51), pursued by Magri (2009), is to propose that implicatures are in the cases such as (51) derived locally instead of globally, that is to say, that the implicatures are derived at the embedded level, below negation, rather than at the matrix level. Assuming together with Magri (2009) grammatical theory of implicature derivation, if implicatures are derived locally in the sentence (51), the resulting meaning of the sentence can be paraphrased in (54a), which is truth-conditionally equivalent to (54b). Granting that it is common knowledge that all Italians come from the same country (even if it is not common knowledge whether Italy is a warm or a cold country), (54a) must then be common knowledge too. In other words, it must be true in all of the worlds of the context set and thus becomes a contextual tautology.

a. It's not the case that some but not all Italians come from a cold country. b. Either all Italians come from a cold country or none of them do.

One can thus explain the deviance of (51): if implicatures are derived locally in (51), the sentence is a contextual tautology.

We can propose an exactly parallel explanation for the deviance of (48). If ignorance inferences are derived locally in (48), the sentence ends up equivalent to (55) (note that the entire conjunction is in the scope of negation).

(55) It is not the case that [both of these girls are Susan or Jane and that the speaker is ignorant about whether or not both of these girls are Susan and that the speaker is ignorant about whether or not both of these girls are Jane].

As it has been pointed out before, given common world knowledge, the speaker cannot be ignorant about the propositions in (56).

a. Both of these girls are Susan.

b. Both of these girls are Mary.

Given that the speaker cannot be ignorant about sentences in (56), (55) is trivially true (it is a contextual tautology). One could thus propose that (48) is deviant because when ignorance inferences are derived locally, it is a contextual tautology. However, while we do have empirical evidence that scalar implicatures can be derived locally in the scope of downward-entailing operators such as negation, such evidence is currently lacking for ignorance inferences. To see that scalar inferences can be derived locally, note that the discourse in (57) can be coherent, especially with a strong focus on some: 
A new case of pragmatically deviant embedded disjunctions

It's not the case that SOME of my students passed: all of them did.

If ignorance inferences can be derived locally, it should be possible to interpret the discourse in (58) as coherent too. It is however not at all clear that this is possible ${ }^{2}$.

(58) ?? It is not the case that both of my closest friends are from France or from Germany — they are both from France.

\subsection{General world knowledge vs. any kind of shared information}

Sentences such as (1) and (3) were proposed to be deviant because they triggered inferences which contradicted common knowledge. However, there seems to be an interaction with where the knowledge comes from, in particular, whether the ignorance inferences triggered by the sentence contradict general world knowledge, or whether they contradict shared information in the context which is not necessarily general world knowledge $)^{3}$. When they contradict information shared in the context which is not general world knowledge (cf. (59b) as a response to (59a)) we seem to get a milder degradation (if any degradation at all) than with (1) and (3).

a. Where are John, Ann, and Bob from originally?

b. ?John isn't French, but all three of them were born in France, Germany, or Spain.

?John wasn't born in France, but all three of them were born in France, Germany, or Spain.

It is not clear why this would be the case. It is possible that ignorance about certain information can be more easily accommodated or dismissed (because, for instance, one can think of a number of scenarios in which the ignorance inferences triggered by a sentence such as (60) would be justified, contrary to the ignorance inferences of (1) and (3)). Clarification of this issue is left for future work.

All three of them were born in France, Germany, or Spain.

\section{Alternative explanation for deviance: inverse scope of disjunction?}

There is (at least) one salient alternative hypothesis for why sentences such as (1) and (3) are deviant, which we will now spell out.

2 This would be an argument for embedded ignorance inferences rather than for embedded distributive inferences because we have seen that unembedded Both of my closest friends are from France or from Germany doesn't seem to trigger distributive inferences.

3 For a discussion on the distinction between the two types of shared knowledge in relation to the deviance of sentences which were discussed in Magri 2009, see Spector 2014. 
Consider the inverse scope interpretation of disjunction in (1), paraphrased in (61a) (and the inverse scope in (3), paraphrased in (61b)). Under the inverse scope interpretation, (1) and (3) are contextual contradictions, again due to the left-uniqueness of the identity copula and the predicate to write.

a. \#Each of those three girls is Mary, or each of those three girls is Susan, or each of those three girls is Jane.

b. \#Each of those three writers wrote Anna Karenina, or each of those three writers wrote Germinal, or each of those three writers wrote Harry Potter.

One could thus hypothesize that (1) and (3) are deviant because under their inverse scope interpretation they are contextual contradictions. There are (at least) three plausible formulations of this hypothesis, as in (62).

(62) Formulation 1: A sentence $S$ is deviant if it is a contextual contradiction under at least one of its scope assignments.

(63) Formulation 2: A sentence $S$ is deviant if it is a contextual contradiction under its preferred scope assignment.

(64) Formulation 3: For a sentence $S$ with two scope assignments $S^{\prime}$ and $S^{\prime \prime}$, the higher the preference for the scope assignment $S^{\prime}$, the more deviant $S$ is perceived to be if under the scope assignment $S^{\prime}$ it is a contextual contradiction.

(62) is clearly too strong. The inverse scope reading of (65a), paraphrased in (65b) is a contextual contradiction, yet (65a) is perfectly acceptable.

(65) a. Exactly three of my cousins gave birth to a boy last year.

b. \#There is a boy such that exactly three cousins of mine gave birth to him last year.

How about the formulations (63) and (64)? Some support for pursuing (63) or (64) comes from the fact that some speakers indeed report a preference for the inverse scope reading of sentences such as (32a), repeated here in (66).

(66) Both of my closest friends are (either) French or Spanish.

While this preference is interesting if it's robust, and merits an explanation, do (63) or (64) provide a satisfactory explanation for the deviance of (1) and (3)?

If (63) or (64) were on the right track to explain the deviance of (1) and (3), the theory of scope ambiguity resolution preferences would have to be to a large extent dissociated from the meaning of the sentence and from the context; otherwise 
A new case of pragmatically deviant embedded disjunctions

it would be difficult to explain why a preferred interpretation of (1) and (3) would be the inverse scope one under which they are contextual contradictions rather than the surface scope one under which they are contingent.

There is however empirical evidence that context and even the meanings of individual words play an important role for the ambiguity resolution of a sentence (see for instance Altmann \& Steedman 1988, a.o.). This is why (63) and (64) might not provide a satisfactory explanation for the deviance of (1) and (3) after all.

Of course, one could argue that we need a much more fine-grained theory on how the discourse context and sentence meaning influence ambiguity resolution. In particular, it could be that some aspects of the discourse context and/or sentence meaning carry more weight than others, and that some aspects, yet to be identified, carry such an important weight that they cannot be overridden even if the preferred scope turns out to be a contextual contradiction because of them. To provide an empirical motivation for such a theory, one could attempt to measure scope preferences for sentences such as (66) and to correlate them with the deviance perceived for sentences such as (1) and (3). Similar tests could be constructed for the cases discussed in Section 7. If indeed one found that there was a correlation there, one could speculate that the precise logical operators in the sentence and their syntactic configuration, as well as the notion of the ratio between the cardinality of the domain of the universal quantifier and the number of disjuncts, play a determining role in ambiguity resolution preferences.

\section{Conclusion}

In this paper we have discussed the surprising deviance of sentences such as (1) and (3). We have proposed that (1) and (3) trigger ignorance inferences which contradict common knowledge, hence their deviance. According to this proposal, ignorance inferences have to be derived blindly to common knowledge, similarly to what has already been proposed for scalar implicatures (Magri 2009). Some empirical challenges remain to be addressed, however. We have also spelled out an alternative account, according to which (1) and (3) are deviant because their inverse scope interpretation is a contextual contradiction. While we didn't provide compelling empirical arguments against the alternative account, we pointed out that it creates a tension with what we know about the ambiguity resolution (Altmann \& Steedman 1988 , a.o.). We have also pointed out some of the experimental tests which could provide further evidence for or against the alternative proposal. 


\section{References}

Altmann, Gerry \& Mark Steedman. 1988. Interaction with context during human sentence processing. Cognition 30(3). 191-238. doi:10.1016/0010-0277(88)900200 .

Bar-Lev, Moshe E. \& Danny Fox. 2017. Universal free choice and innocent inclusion. doi:10.3765/salt.v27i0.4133. SALT 2017.

Buccola, Brian \& Andreas Haida. 2018. Obligatory irrelevance and the computation of ignorance inferences. Available at https://ling.auf.net/lingbuzz/003600.

Chierchia, Gennaro. 2013. Logic in Grammar. Oxford University Press. doi:10.1093/acprof:oso/9780199697977.001.0001.

Chierchia, Gennaro, Danny Fox \& Benjamin Spector. 2008. The grammatical view of scalar implicatures and the relationship between semantics and pragmatics. Semantics: An International Handbook of Natural Language Meaning .

Crnič, Luka, Emmanuel Chemla \& Danny Fox. 2015. Scalar implicatures of embedded disjunction. Natural Language Semantics 23(4). 271-305. doi:10.1007/s11050-015-9116-x.

Denić, Milica. 2018. Cardinality effects on ignorance inferences of disjunction in quantified sentences. SuB 2018 presentation.

Fox, Danny. 2007. Free choice and the theory of scalar implicatures. In Presupposition and implicature in compositional semantics, 71-120. Springer. doi:10.1057/9780230210752_4.

Fox, Danny \& Roni Katzir. 2011. On the characterization of alternatives. Natural language semantics 19(1). 87-107. doi:10.1007/s11050-010-9065-3.

Gazdar, Gerald. 1980. Pragmatics and logical form. Journal of Pragmatics doi:10.1016/0378-2166(80)90014-4.

Grice, H. Paul. 1975. Logic and conversation, 41-58. New York: Academic Press.

Katzir, Roni. 2007. Structurally-defined alternatives. Linguistics and Philosophy 30(6). 669-690. doi:10.1007/s10988-008-9029-y.

Kaufmann, Stefan, Cleo Condoravdi \& Valentina Harizanov. 2006. Formal approaches to modality. The expression of modality 71-106.

Klinedinst, Nathan Winter. 2007. Plurality and possibility. Los Angeles, California: University of California Los Angeles dissertation.

Magri, Giorgio. 2009. A theory of individual-level predicates based on blind mandatory scalar implicatures. Natural language semantics 17(3). 245-297. doi:10.1007/s11050-009-9042-x.

Meyer, Marie-Christine. 2013. Ignorance and grammar. Cambridge, Massachusetts: Massachusetts Institute of Technology dissertation.

Meyer, Marie-Christine. 2014. Deriving Hurford's constraint. In Semantics and linguistic theory, vol. 24, 577-596. doi:10.3765/salt.v24i0.2518. 
A new case of pragmatically deviant embedded disjunctions

Sauerland, Uli. 2004. Scalar implicatures in complex sentences. Linguistics and philosophy 27(3). 367-391. doi:10.1023/B:LING.0000023378.71748.db.

Spector, Benjamin. 2006. Aspects de la pragmatique des opérateurs logiques. Paris, France: University of Paris 7 dissertation.

Spector, Benjamin. 2014. Scalar implicatures, blindness and common knowledge: Comments on Magri (2011). In Pragmatics, semantics and the case of scalar implicatures, 146-169. Springer. doi:10.1057/9781137333285_6.

Spector, Benjamin. 2018. What do we learn from game-theoretic pragmatics? SALT 28 presentation.

Stalnaker, Robert. 2002. Common ground. Linguistics and philosophy 25(5-6). 701-721. doi:10.1023/A:1020867916902.

Milica Denić

LSCP \& IJN

École Normale Supérieure

29 rue d'Ulm, 75005 Paris, France

milica.denic@ens.fr 